\title{
Towards General Algorithms for Grammatical Inference $^{\star}$
}

\author{
Alexander Clark \\ Department of Computer Science \\ Royal Holloway, University of London \\ alexc@cs.rhul.ac.uk
}

\begin{abstract}
Many algorithms for grammatical inference can be viewed as instances of a more general algorithm which maintains a set of primitive elements, which distributionally define sets of strings, and a set of features or tests that constrain various inference rules. Using this general framework, which we cast as a process of logical inference, we re-analyse Angluin's famous LSTAR algorithm and several recent algorithms for the inference of context-free grammars and multiple context-free grammars. Finally, to illustrate the advantages of this approach, we extend it to the inference of functional transductions from positive data only, and we present a new algorithm for the inference of finite state transducers.
\end{abstract}

* The full version of this paper is published in the Proceedings of the 21th International Conference on Algorithmic Learning Theory, Lecture Notes in Artificial Intelligence Vol. 6331 . 